\title{
Real-time measurement on deformation fields of notched samples under impact tension
}

\author{
Chenguang Huang ${ }^{\mathrm{a}, *}$, Siying Chen ${ }^{\mathrm{b}}$, Zhuping Duan ${ }^{\mathrm{a}}$ \\ ${ }^{a}$ National Key Laboratory of Nonlinear Mechanics, Institute of Mechanics, Chinese Academy of Sciences, \\ Beijing 100080, People's Republic of China \\ ${ }^{\mathrm{b}}$ Photoelectricity Department, Beijing Institute of Technology, Beijing 100081, People's Republic of China
}

Received 11 August 2003; received in revised form 12 January 2004; accepted 14 January 2004

\begin{abstract}
In this paper, a real-time and in situ optical measuring system is reported to observe high-velocity deformations of samples subjected to impact loading. The system consists of a high-speed camera, a $\mathrm{He}-\mathrm{Ne}$ laser, a frame grabber, a synchronization device and analysis software based on digital correlation theory. The optical system has been adapted to investigate the dynamic deformation field and its evolution in notched samples loaded by an split Hopkinson tension bar, with a resolution of $50 \mu \mathrm{m}$ and an accuracy of $0.5 \mu \mathrm{m}$. Results obtained in experiments are discussed and compared with numerical simulations. It is shown that the measuring system is effective and valid.
\end{abstract}

(C) 2004 Elsevier Ltd. All rights reserved.

Keywords: Hopkinson bar; Optical measurement; Digital correlation method

\section{Introduction}

The split Hopkinson pressure/tension bar (SHPB/SHTB) is widely used to produce highvelocity tensile deformation, and to investigate this material dynamic behavior. However, details of the local deformation in samples are left unknown, because it is presumed in the classic SHTB method that the stress and strain are uniform over the specimen. This deficiency hinders the experimental study of some typical impact problems by Hopkinson devices, such as deformation localization and damage evolution and interactions.

Compared with traditional electric measuring method, such as strain gauges, optical testing technology enjoys the advantages of non-contact and extremely fast response. And the measuring

\footnotetext{
${ }^{*}$ Corresponding author.

E-mail address: huangcg@imech.ac.cn (C. Huang).
} 
accuracy can be half the wavelength of the laser light or even shorter. At the same time, the spatial resolution can be easily to a micron. So, more and more scientists are trying to explore impact deformation by using advanced optical methods. The first attempt to measure transient deformations using a ruby laser was carried out by Cookson et al. in 1978 [1]. As desk-top computers with digital image processing capabilities were not available at that time, speckle interferograms were recorded using a tape recorder and fringe patterns were obtained by electronic processing. Later, digital speckle pattern interferometry (DSPI) was applied to the measurement of in-plane deformation of rotating objects [2]. More recently, Tong et al. reported a technique for measuring in-plane displacement in a plate under impact loading [3]. In recent years, some similar investigations have been published [4-13].

However, real time and in situ observations of impact deformation and failure have not been implemented, because all the above work used stroboscopic or pulsed-laser illumination, and obtained only one image in one specimens. In order to study the deformation evolution, a series of samples were tested under the same loading conditions, and it was hypothesized that all specimen and loading conditions would be absolutely uniform.

In this paper, we propose a transient optical measuring system, based on the digital speckle correlation method (DSCM). The dynamic deformation patterns and their temporal evolution for notched LY12 samples under impact loadings were recorded, the time-interval between each image being $10 \mu \mathrm{s}$. In the following sections, the elements of the measuring system, fundamental theory and the experimental results are shown in detail.

\section{Basic theory and measurement system}

If $u, v, w$ denote the displacements in the $x, y, z$ directions, respectively, and a line $P Q(\mathrm{~d} x, \mathrm{~d} y, \mathrm{~d} z)$ with end points $P(x, y, z)$ and $Q(x+\mathrm{d} x, y+\mathrm{d} y, z+\mathrm{d} z)$ is changed into $P^{*} Q^{*}\left(\mathrm{~d} x^{*}, \mathrm{~d} y^{*}, \mathrm{~d} z^{*}\right)$ after deformation. Then, we can get [14]

$$
\begin{aligned}
& P^{*}=\left(x^{*}, y^{*}, z^{*}\right)=[x+u(P), y+v(P), z+w(P)] \\
& Q^{*}=\left(x+u(P)+\mathrm{d} x^{*}, y+v(P)+\mathrm{d} y^{*}, z+w(P)+\mathrm{d} z^{*}\right), \\
& \mathrm{d} x^{*} \cong\left(1+\frac{\partial u}{\partial x}\right) \mathrm{d} x+\left(\frac{\partial u}{\partial y}\right) \mathrm{d} y+\left(\frac{\partial u}{\partial z}\right) \mathrm{d} z \\
& \mathrm{~d} y^{*} \cong\left(1+\frac{\partial v}{\partial y}\right) \mathrm{d} y+\left(\frac{\partial v}{\partial x}\right) \mathrm{d} x+\left(\frac{\partial v}{\partial z}\right) \mathrm{d} z, \\
& \mathrm{~d} z^{*} \cong\left(1+\frac{\partial w}{\partial z}\right) \mathrm{d} z+\left(\frac{\partial w}{\partial x}\right) \mathrm{d} x+\left(\frac{\partial w}{\partial y}\right) \mathrm{d} y .
\end{aligned}
$$

The lengths of the line before and after deformation are

$$
\begin{aligned}
& |P Q|^{2}=(\mathrm{d} x)^{2}+(\mathrm{d} y)^{2}+(\mathrm{d} z)^{2}, \\
& \left|P^{*} Q^{*}\right|^{2}=\left(\mathrm{d} x^{*}\right)^{2}+\left(\mathrm{d} y^{*}\right)^{2}+\left(\mathrm{d} z^{*}\right)^{2} .
\end{aligned}
$$

If the displacement and its gradient are postulated for a point in the initial configuration, the deformed infinitesimal area around the point in the current configuration can be obtained through 
Eqs. (1)-(3), and is called the virtual area. The correlation coefficient of gray levels between the initial infinitesimal area and the virtual area is calculated by using Eq. (4). When the correlation coefficient arrives at the maximum value through use of an appropriate iteration algorithm, the supposed displacement and its gradient can be accepted as the true ones. That is to say, if we can record the surface gray pictures of deformed object at two different times, the deformation field can be deduced by the correlation method.

$$
C\left(u, v, \frac{\partial u}{\partial x}, \frac{\partial u}{\partial y}, \frac{\partial v}{\partial x}, \frac{\partial v}{\partial y}\right)=\frac{\sum\left[F(x, y) \cdot G\left(x^{*}, y^{*}\right)\right]}{\left[\sum\left(F(x, y)^{2}\right) \cdot \sum\left(G\left(x^{*}, y^{*}\right)^{2}\right)\right]^{1 / 2}}=\frac{\langle F \cdot G\rangle}{\left[\left\langle F^{2}\right\rangle \cdot\left\langle G^{2}\right\rangle\right]^{1 / 2}},
$$

where $F(x, y)$ is the gray function of the first image while $G\left(x^{*}, y^{*}\right)$ denotes that after deformation (Fig. 1).

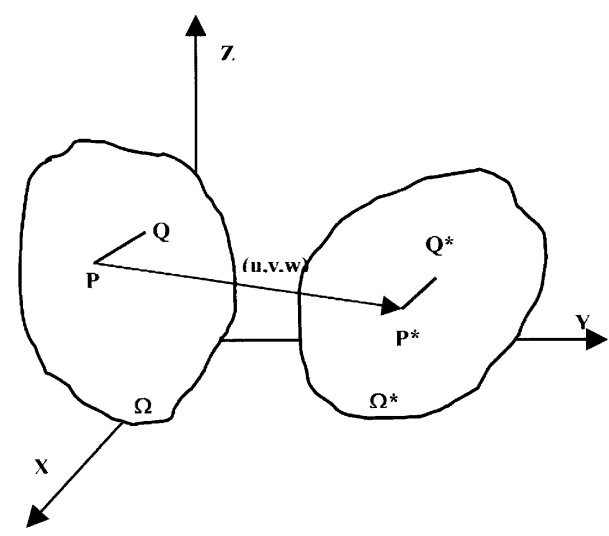

Fig. 1. Deformation and motion of object.

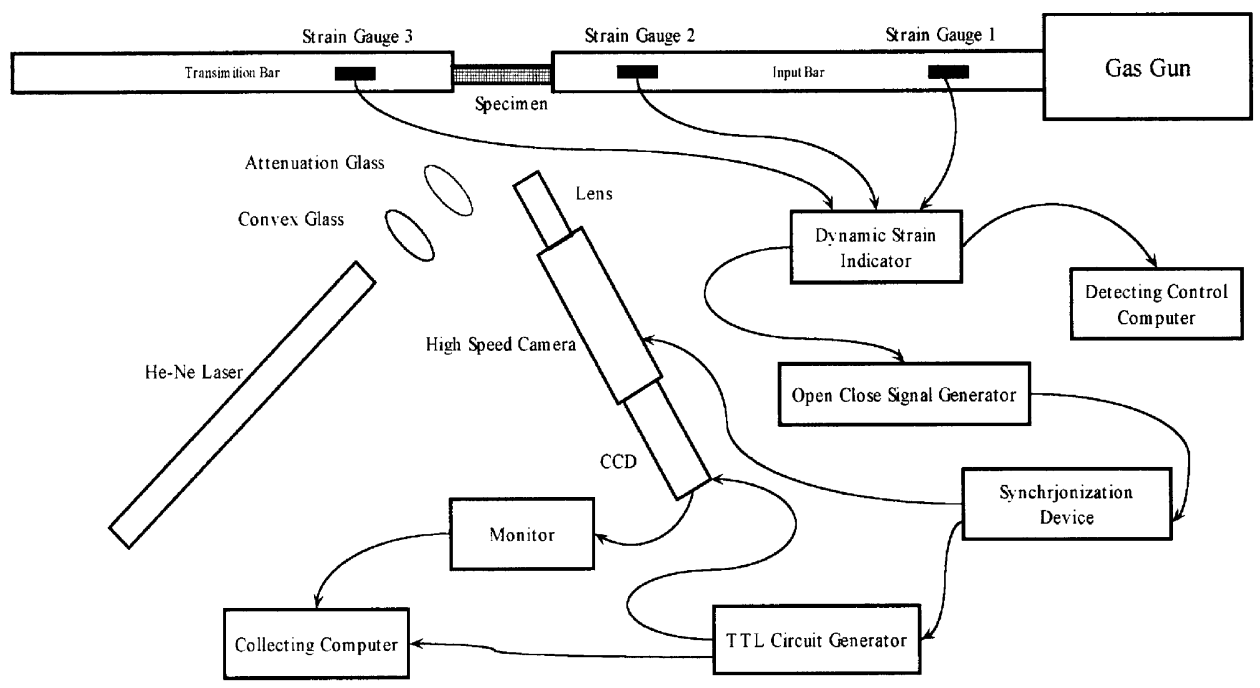

Fig. 2. Sketch of measuring system. 


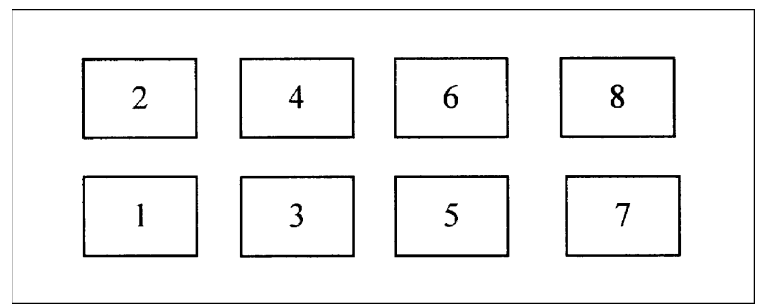

Fig. 3. High-speed camera imaging sequence.

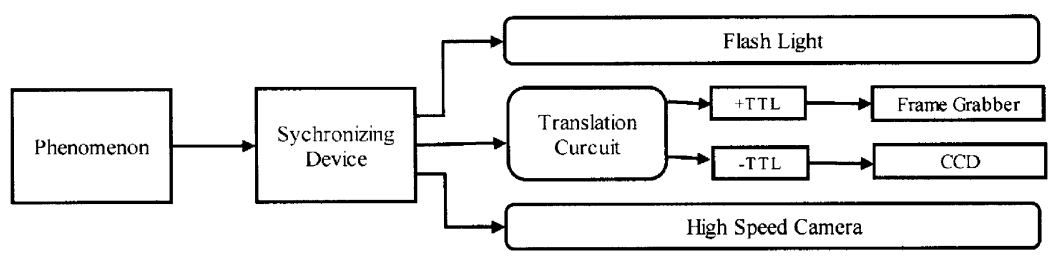

Fig. 4. Synchronizing control.

The measuring system, shown in Fig. 2, consists of a high-speed camera, a He-Ne laser, synchronization and recording devices.

Every print obtained by the high-speed camera is divided into 8 smaller images with the sequence depicted in Fig. 3, with a time interval of $10 \mu$ s.

The high-speed camera, IMACON 790, is improved by using the MTV-1881EX CCD as a recorder instead of Polylad films. This CCD is produced by MITON company and has a resolution of $795(H) \times 596(\mathrm{~V})$ and an image area of $7.95 \mathrm{~mm} \times 5.08 \mathrm{~mm}$. The minimum sensitive illumination is $0.02 \mathrm{~lx}$ and the signal-to-noise performance is greater than $48 \mathrm{db}$.

Synchronizing control is important and complicated in dynamic measurements. The strain signal, which comes from a strain gauge attached to the input bar, is used as the trigger source. A three-channel synchronization device with accurate time-delay is designed to trigger the flash, high-speed camera and frame grabber in sequence. The sketch map of synchronizing control is shown in Fig. 4.

\section{Experimental preparation}

The impacting experiments were performed in an SHTB, which was built in the Institute of Mechanics, the Chinese Academy of Sciences.

Before the experiments, static and dynamic verifications are conducted with a common plate tensile sample (shown in Fig. 5), on the surface of which, the grid of $2 \mathrm{lp} / \mathrm{mm}$ is engraved by a mechanical method.

In the static verification, the SHTB is not operated so the sample remains static, an artificial signature is used as a trigger. In this verification, the devices status, the length scale and the system gray error in the high-speed camera image can be obtained. The dynamic verification is performed 
to check the running conditions of the SHTB and synchronizing controlling system. Fig. 6 shows the images taken in the two kinds of verification.

The notched sample is made of an aluminium alloy named LY12 and its surface is mildly polished to increase the intensity of reflected light so that a good exposure can be obtained. Notches with a depth of $1 \mathrm{~mm}$, are cut symmetrically in the middle of the specimen by a linear cutting machine, as shown in Fig. 7.

To satisfy the exposure requirements the camera is not perpendicular to the sample surface hence the slope angle has to be adjusted in image processing. The image resolution ratio is about $50 \mu \mathrm{m}$ and the accuracy of measurement displacement is $0.5 \mu \mathrm{m}$. The accuracy of measuring

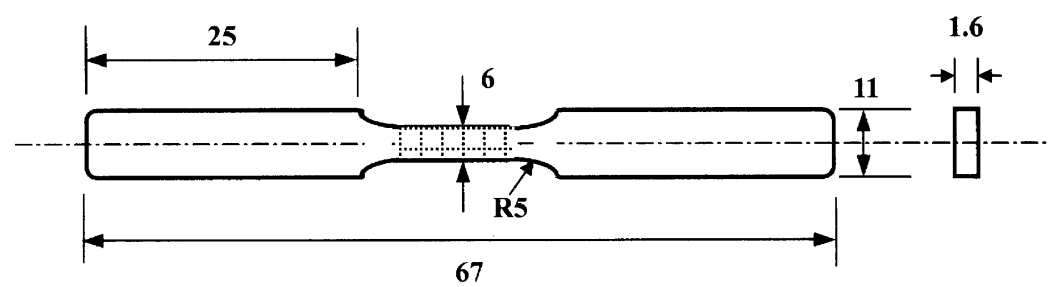

Fig. 5. Dimensions of impact tensile sample.

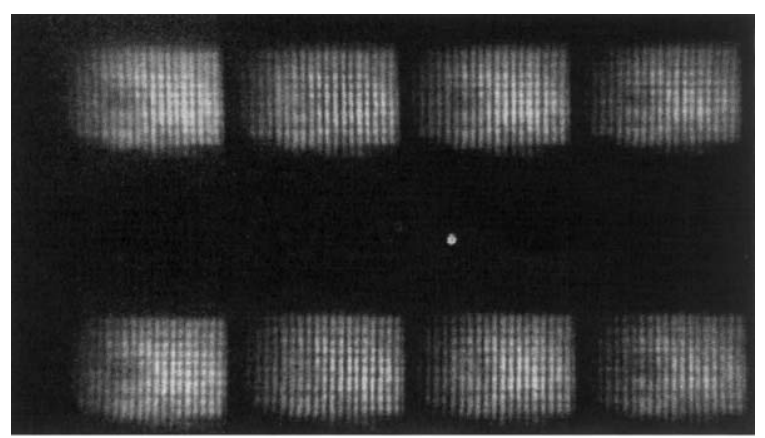

(a)

Image of static verification

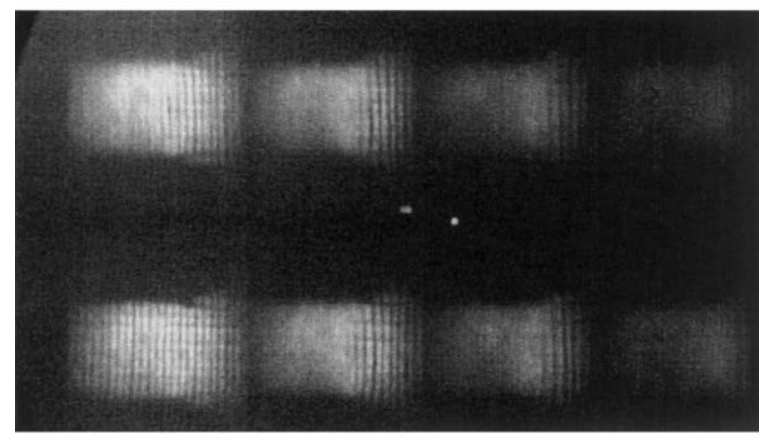

(b)

Image of dynamic verification

Fig. 6. (a) Image of static verification and (b) image of dynamic verification. 
displacement is determined by the image-processing code design, and can reach the one percent of the size of single pixel. While the image resolution is determined by the structure of the imageforming system, mainly including the high-speed camera and the CCD.

The image processing includes mainly three steps, as follows:

1. Image cutting. Because each CCD photograph consists of 8 small pictures, which depict the object at different times, a method must be employed to divide the photograph accurately. In this paper, a method based on correlation calculation is presented, and it is used in a specially written code with MATLAB v6.0. When we select an interesting region in the first small picture, the other seven pictures can be obtained automatically and saved as different files. Because the successive pictures are defined by the codes based on Statistic Correlation Method, it can be considered that the rigid displacement has been erased in the area selection.

2. Image pre-processing. In this step, the gray-degree difference and rigid motion among eight small pictures resulted from measuring system error are taken out.

3. Displacement calculating. The calculation is based on the correlation iteration, as depicted in the above equations.

\section{Experimental results and discussions}

In Fig. 8, the dynamic deformation of the notched specimen under impact tension is shown. The small square is the calculating area near the notch. From this figure, we can find directly the

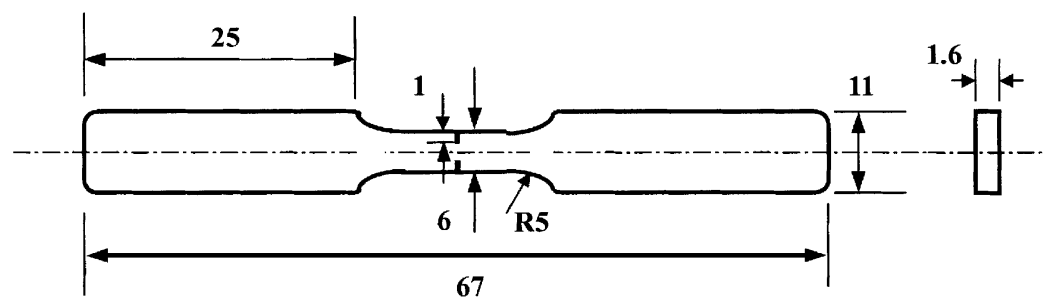

Fig. 7. Dimensions of notched sample.

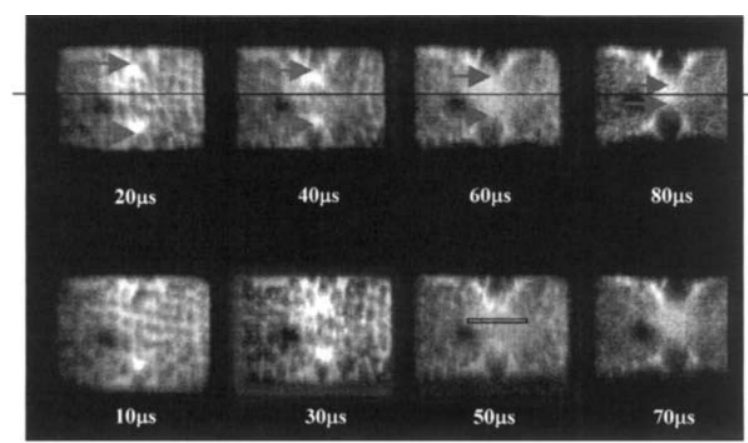

Deformation processing of notched sample under impacting tension

Fig. 8. Deformation processing of notched sample under impact tension. 
deformation pattern and evolution on the macroscopic scale. The deformation concentrates in the neighbourhood of the notches, and the two notches are closed gradually. Quantitative displacement fields with high resolution can just be gained by the correlation analysis.
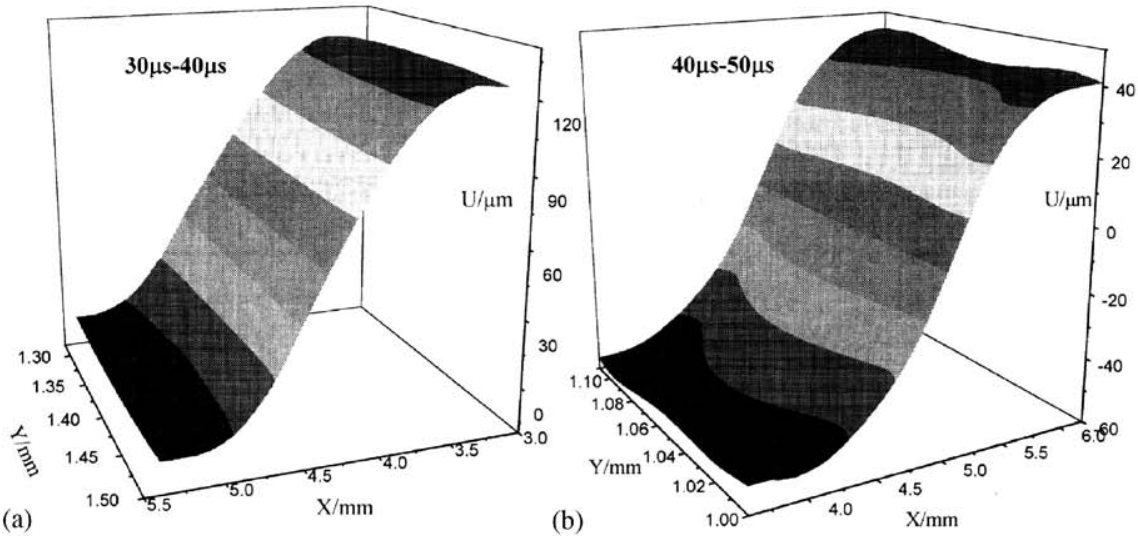

Displacement along tensile direction (a-b)
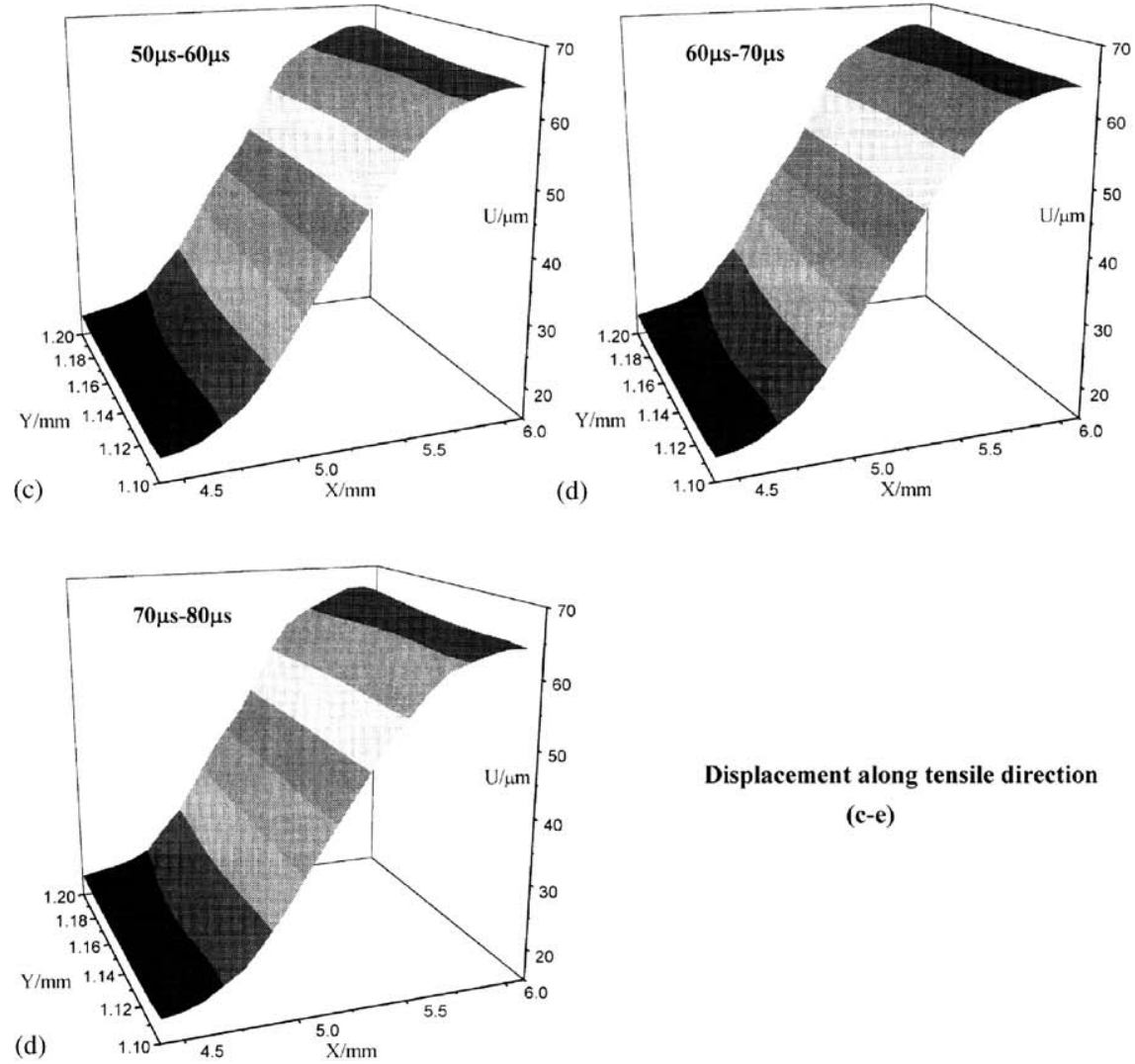

Displacement along tensile direction

(c-e)

Fig. 9. Displacement along tensile direction. 
Fig. 9 shows the distribution of $u$, the displacement component in the tensile direction, at different times. It must be noted, the displacement is obtained with reference to the configuration of the former dynamic image, not the static sequence, and rigid displacement is eliminated properly.

From Fig. 9, we can find that the distribution of $u$ is not linear. In the middle region, the gradient of displacement is very steep, but at the two ends of the observed region, the change of displacement is much more gradual. As we know, a steep displacement gradient means a large strain. All the experimental results exhibit similar regularity and are reasonable.

To confirm the experimental observations, numerical simulations of the impact tension process for a notched specimen were conducted using the commercial FEM code LS-DYNA. Fig. 10 shows the distribution of $u$ along the central line of the specimens, from 40 to $50 \mu \mathrm{s}$. Comparing the experimental result (Fig. 9b) with the numerical simulation, we find that the in situ experimental observations of the dynamic deformation are valid.

Now, the strain field can be determined by using basic continuum mechanics theory,

$$
\begin{aligned}
& E_{\mathrm{IJ}}=\frac{1}{2}\left(C_{\mathrm{IJ}}-1\right), \\
& C_{\mathrm{IJ}}=F_{\mathrm{iI}}^{\mathrm{T}} F_{\mathrm{iJ}}, \\
& F_{\mathrm{iI}}=\frac{\partial x_{\mathrm{i}}}{\partial X_{\mathrm{I}}}=\delta_{\mathrm{iI}}+\frac{\partial u_{\mathrm{i}}}{\partial X_{\mathrm{I}}},
\end{aligned}
$$

where $\boldsymbol{E}, \boldsymbol{C}, \boldsymbol{F}$ denote the Green strain tensor, deformation tensor and deformation gradient tensor, respectively.

For the deformation field, shown in Fig. 9b, the normal strain in the tensile direction, the shear strain and equivalent strain are obtained and shown in Fig. 11.

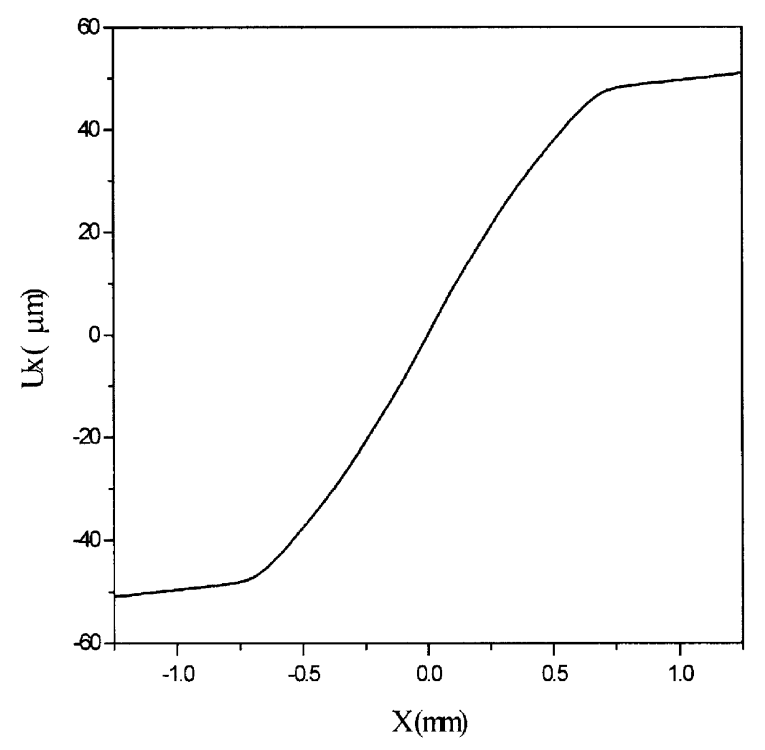

Fig. 10. Numerical results for displacement field. 

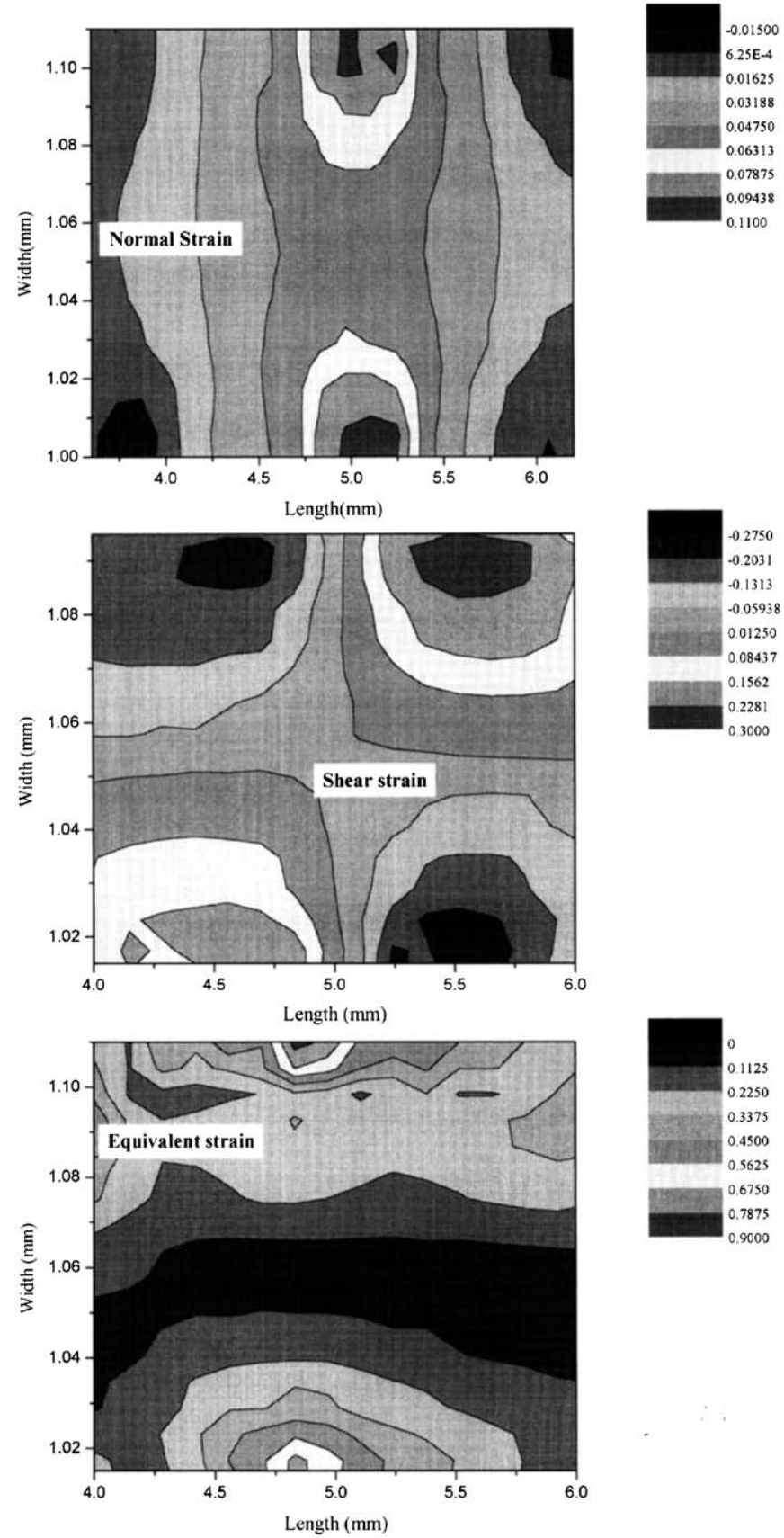

Fig. 11. Strain fields in notched samples. 


\section{Concluding remarks}

In this paper, DSCM is developed and applied to investigate the high-velocity deformation of a notched sample loaded under impact tension. The main results include,

1. A high velocity DSCM measuring system has been built, including analysis software based on correlation calculation and iteration theory.

2. Dynamic and static verifications are conducted to check the running conditions of the various devices and control system.

3. The deformation field and its evolution for notched samples loaded in impact were obtained experimentally with a resolution of $50 \mu \mathrm{m}$ and an accuracy of $0.5 \mu \mathrm{m}$. The experimental observations are reasonable and fit the numerical simulation quantitatively.

4. The resolution and degree of accuracy could be improved by using a more advanced lens and CCD, and the proper treatment of sample surfaces.

5. The correlation iteration algorithm should be upgraded to speed up the calculation process and to reduce errors.

\section{Acknowledgements}

The authors thank the National Natural Science Foundation of China (10002021) The Special Funds for Major State Basic Research Project (2002CB412706) and the National High Technology Planning Project for financial support.

\section{References}

[1] Cookson TJ, Butters JN, Pollad HC. Pulse lasers in electronic speckle pattern interferometry. Opt Laser Tech 1978;10:119-24.

[2] Preater RWT. Analysis of rotating component strains using electronic speckle pattern interferometry. Proc SPIE 1984;473:40-3.

[3] Tong J, Zhang D, Li H, Li L. Study on in-plane displacement measurement under impact loading using digital speckle pattern interferometry. Opt Eng 1996;35:1080-3.

[4] Fernandez A, Moore AJ, Perez Lopez C, Doval AF, Blanco-Garcia J. Study of transmit deformations with pulsed TV holography: application to crack detection. Appl Opt 1997;36:2058-65.

[5] Pedrini G, Pfister B, Tiziani HJ. Double pulse-electronic speckle interferometry. J Mod Opt 1993;40:89-96.

[6] Kaufmann GH, Diaz FV, Galizzi GE, Moore AJ. Transient in-plane deformation analysis by means of pulsed TV holography. Optik 1998;108:43-7.

[7] Moore AJ, Hand DP, Barton JS, Jones JDC. Transient deformation measurement with electronic speckle pattern interferometry and a high-speed camera. Appl Opt 1999;38:1159-62.

[8] Fernandez A, Doval AF, Davila A, Blanco-Garcia J, Perez-Lopez C, Fernandez JL. Double-pulsed carrier speckle-shearing pattern interferometry for transient deformation analysis. Proc SPIE 1998;3478:353-8.

[9] Farrant DI, Kaufmann GH, Petzing JN, Tyrer JR, Oreb BF, Kerr D. Transient deformation measurement using dual-pulse addition ESPI. Appl Opt 1998;37:7259-67.

[10] Ruiz PT, Kaufmann GH, Moller O, Galizzi GE. Evaluation of impact-induced transient deformations using double-pulsed electronic speckle pattern interferometry and finite elements. Optics Lasers Eng 2000;32:473-84.

[11] Huntley JM. Special issue on measurements of solid deformation by phase-shifting interferometry. Optics Lasers Eng 1996;24(5):363-4. 
[12] Melin LG, Goldrein HT, Huntley JM. A study of mode-I deformation cracks by high-magnification moiré interferometry. Composites Sci Technol 1998;58(3):515-25.

[13] Davila A, Ruiz PD, Kaufmann GH, Huntley JM. Measurement of sub-surface delaminations in carbon fibre composites using high-speed phase-shifted speckle interferometry and temporal phase unwrapping. Optics Lasers Eng 2003;40(5):447-58.

[14] Jin GC. Computer-aided optical metrology. 1997. 